Original Article

\title{
Two Different Minimally Invasive Techniques for Female Patients with Atrial Septal Defects: Totally Thoracoscopic Technique and Right Anterolateral Thoracotomy Technique
}

\author{
Ming Xu, MD, Shaoping Zhu, MD, Xianguo Wang, MD, Hua Huang, PhD,
} and Jinping Zhao, MD, PhD

\begin{abstract}
Background: To compare the outcomes of totally thoracoscopic technique (TTS) vs. right anterolateral thoracotomy technique (RALT) in female patients undergoing minimal invasive atrial septal defect (ASD) correction.

Methods: From March 2011 to January 2013, 125 female patients underwent minimally invasive atrial septal defect closure, of whom 62 patients were in the TTS group and 63 were in the RALT group.

Results: Procedures were performed successfully in all patients without in-hospital mortality or major complications. cardiopulmonary bypass (CPB) time were $48.95 \pm 15.63 \mathrm{~min}$ in TTS group, $31.4 \pm 8.04 \mathrm{~min}$ in RALT group $(p<0.001)$; the cross-clamp time were $26.92 \pm 11.84 \mathrm{~min}$ in TTS group and $18.51 \pm 6.11 \mathrm{~min}$ in RALT group $(p<0.001)$. The length of incision in RALT group $(6.02 \pm 1.03 \mathrm{~cm})$ was longer than TTS group $(5.31 \pm 0.68 \mathrm{~cm})$ and the difference was significant $(\mathbf{p}<\mathbf{0 . 0 0 1})$. The overall satisfaction rate for the cosmetic results of TTS was $100 \%$ and was $96.83 \%$ (61/63 patients) in RALT patients. During follow-up, all patients in TTS group were satisfied expect two patients complained that scar was too long at groin. Reasons for a lower score in RALT group included the long scar in the chest; a RALT incision that was located too medially (coming off the bra line) and asymmetrical breast development. Conclusions: Both TTS and RALT are valid and reliable cosmetic surgical techniques for repairing ASDs in female patients. Both techniques allow excellent cosmetic and functional results in most female patients. The totally thoracoscopic technique may gain shorter incision and cosmetic results compared with RALT.
\end{abstract}

Keywords: atrial septal defect, minimally invasive surgery, thoracoscopy, anterolateral thoracotomy

\section{Introduction}

Atrial septal defect (ASD) is one of the most common congenital heart diseases. Although transcatheter closure

Department of Thoracic and Cardiovascular Surgery, Zhongnan Hospital of Wuhan University, Wuhan, Hubei 430071, P.R.China

Received: January 6, 2015; Accepted: February 27, 2015

Corresponding author: Jinping Zhao, MD, PhD. Department of Thoracic and Cardiovascular Surgery, Zhongnan Hospital of Wuhan University, 169 Donghu Road, Wuhan, Hubei, 430071, P.R.China Email: researchcn@ hotmail.com

(C)2015 The Editorial Committee of Annals of Thoracic and Cardiovascular Surgery. All rights reserved. of atrial septal defects is standard practice and excellent results have been reported with low early and late complication rates, ${ }^{1)}$ primary surgical repairs are still needed for large secundum defects with limited septal margins and complex ASDs including ostium primum, sinus venosus ASDs as well as fenestrated or aneurysmal interatrial septal. In recent years, cosmetic and psychologic implications of cardiac surgery have assumed increasing importance, especially female patients. As a result, a variety of minimally invasive cardiac surgical techniques have been developed, including right thoracotomy, ${ }^{2-6)}$ and video-assisted methods. ${ }^{7)}$ 
Table 1 Clinical characteristics and perioperative data

\begin{tabular}{|c|c|c|c|c|}
\hline Variables & TTS Group $(n=62)$ & RALT Group $(n=63)$ & $\mathrm{t} / \chi^{2}$ value & $\mathrm{p}$ value \\
\hline Mean age & $29.37 \pm 11.6$ & $24.14 \pm 15.1$ & 2.169 & 0.032 \\
\hline Weight (kg) & $48.85 \pm 14.93$ & $43.13 \pm 16.93$ & 2 & 0.048 \\
\hline Qp/Qs & $2.37 \pm 0.38$ & $2.25 \pm 0.42$ & 1.641 & 0.103 \\
\hline Size of defect $(\mathrm{cm})$ & $2.6 \pm 0.79$ & $2.74 \pm 0.74$ & 0.997 & 0.321 \\
\hline CPB time(min) & $48.95 \pm 15.63$ & $31.4 \pm 8.04$ & 7.878 & $<0.001$ \\
\hline Cross-clamp time(min) & $26.92 \pm 11.84$ & $18.51 \pm 6.11$ & 4.981 & $<0.001$ \\
\hline Drainage (mL) & $176.5 \pm 94.2$ & $202.9 \pm 75.9$ & 1.648 & 0.087 \\
\hline Intubation time (h) & $6.25 \pm 3.09$ & $7.36 \pm 3.2$ & 1.975 & 0.051 \\
\hline ICU stay (h) & $23.52 \pm 7.68$ & $26.6 \pm 8.31$ & 2.167 & 0.032 \\
\hline Incision length $(\mathrm{cm})$ & $5.31 \pm 0.68$ & $6.02 \pm 1.03$ & 4.579 & $<0.001$ \\
\hline Hospital stay (days) & $5.29 \pm 1.42$ & $6.27 \pm 1.68$ & 3.503 & 0.001 \\
\hline \multicolumn{5}{|l|}{ Complications } \\
\hline Reoperation & 0 & $1(1.59 \%)$ & - & NS \\
\hline Wound infection & 0 & $1(1.59 \%)$ & - & NS \\
\hline Postoperative pneumothorax & $2(3.23 \%)$ & $4(6.35 \%)$ & 1.526 & 0.217 \\
\hline Mortality & 0 & 0 & - & - \\
\hline
\end{tabular}

TTS: totally thoracoscopic technique; RALT: right anterolateral thoracotomy technique

In our center, right anterolateral thoracotomy (RALT) and totally thoracoscopic technique (TTS) have been employed to repair atrial septal defect for several years. This retrospective study reviews our results and experiences with the TTS technique and RALT technique for correction of atrial septal defect, focusing on patient satisfaction with the cosmetic result of both different techniques.

\section{Materials and Methods}

This study was approved by the Institutional Review Board of Wuhan University and was in compliance with Health Insurance Portability and Accountability Act regulations and the Declaration of Helsinki. The written informed consent and operation related permission, including the use of their pictures, were obtained from each patient, parent, or guardian.

Between March 2011 and January 2013, 125 female patients underwent elective ASD closure. In accordance with the patient's preference, and after discussion in our cardiologic/surgical conference, surgical access was made totally thoracoscopic technique (TTS Group, $n=62$ ), right anterolateral thoracotomy (RALT Group, $\mathrm{n}=63$ ). All patients underwent ASD closure of by the same surgical team. The demographic data of the two groups were listed in Table 1.

A satisfaction score was collected from each patient; it ranged from 0 to 5 and was assigned considering both the clinical and the cosmetic results of the TTS and RALT approach. A score of 5 was judged as an excellent result, 3 to 4 as good, and 0 to 2 as unsatisfactory.

\section{Operative Technique}

After induction of general anesthesia, a double-lumen endotracheal tube was placed to allow for single-lung ventilation in all the patients. The respiration rate was set between 18 and 30 breaths/min, and the arterial oxygen saturation rate was maintained at greater than $97 \%$. A transesophageal echocardiograph probe was inserted routinely in all patients to assess the place of the venous cannulations and the surgical result as well as to detect possible intracardiac air.

\section{In TTS Group}

Patients were positioned in supine position with right side of the body elevated to $15^{\circ}$ to $20^{\circ}$. The cardiopulmonary bypass was established by peripheral arterial and venous cannulating. Three $1-\mathrm{cm}$ to $2-\mathrm{cm}$ incisions were made in the fourth intercostal space on the right side of the sternum, in the sixth intercostal space on a midclavicular line, and in the fifth intercostal space on the right midaxillary line, respectively. These incisions allow the entry of tissue forceps or suture needles, scissors, and endoscopic camera or thoracoscope (Fig. 1).

Pericardiotomy was performed and 3 or 4 sutures were placed to suspend the pericardium. Caval snares were placed in the superior and inferior vena cava to install total cardiopulmonary bypass. $\mathrm{CO}_{2}$ was converied to the operative field through the third incision. A long perfusion needle was inserted in the aorta through the pursestring suture with the help of the thoracoscope. And when core body temperature was reduced to $32^{\circ} \mathrm{C}$ and an 


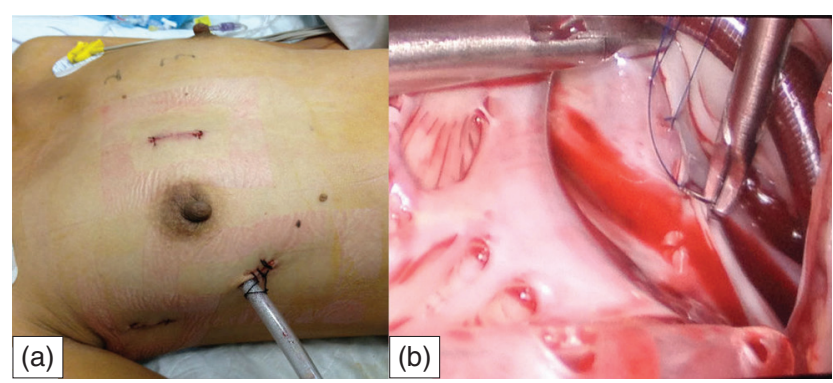

Fig. 1 Totally thoracoscopic technique: three incisions were made on the right chest wall (a) and an intraoperative view of atrial septal defect (ASD) that was closed by sutures (b).

aortic crossclamp was introduced through the third incision and occluded the ascending aorta under the direct view of thoracoscopy. After cardiac asystolic arrest with anterograde cold cardioplegia, the right atriotomy was opened from a site parallel to the atrioventricular annulus by the forceps and scissors through the incision 2 and incision 3 respectively, and four sutures were placed on the incision to expose the intra-atrial structure. The atrial septal defect type, size, and relation to the atrial structures were identified carefully. The ASD was closed with 4-0 prolene directly or with a Dacron patch based on the size of the defect. Deairing was done from the perfusion cannula and the ASD defect before knotting by lung inflation. The right atrium was sutured by 4-0 prolene after the aortic clamp was released and body been warmed. $\mathrm{CO}_{2}$ was removed after the close of right atrium. The cannula was removed after weaning from cardiopulmonary bypass. A chest tube was placed from incision 1 to drain effusion and air (Fig. 1).

\section{In RALT Group}

In patients who underwent RALT approach, a semilunar incision was made in the sulcus of the right breast (Fig. 2a), entering the chest at the fourth intercostal space. ${ }^{2,8)}$ Particular care was necessary in female patients especially the prepubescent patients to avoid any possible future interference with breast development. The length of the incision was approximately $5 \mathrm{~cm}$ to $9 \mathrm{~cm}$ depending on patient's physical characteristics and the type of lesion being treated. The thoracic cavity was entered through the fourth intercostal space. The lung was retracted posteriorly using wet sponges to expose the pericardium. Pericardiotomy was performed to provide enough exposure of the ascending aorta and inferior vena cava. After heparin sodium administration, the

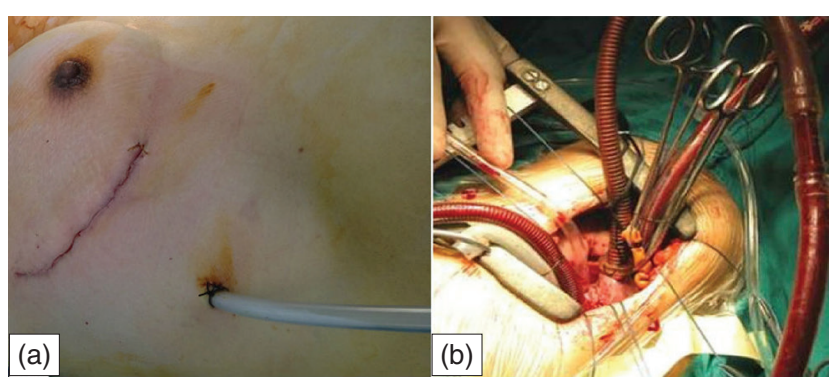

Fig. 2 Right anterolateral thoracotomy technique (RALT): a semilunar incision was made in the sulcus of the right breast, entering the chest at the fourth intercostal space (a) and cardiopulmonary bypass was established by cannulating of aorta, superior vena cava and IVC (b).

aorta was cannulated with the help of two long vascular clamps. One clamp drawn the cannulation site down, and the other held the top of the aortic cannula to push it in place. Then the superior vena cava and inferior vena cava are cannulated. Cardiopulmonary bypass with mild hypothermia $\left(32^{\circ} \mathrm{C}\right)$ is instituted (Fig. 2). An aortic perfusion needle was inserted in the aorta through the pursestring suture. And then, the aorta was cross-clamped and cold blood cardioplegic solution was used for myocardial protection.

After snaring of the superior and inferior vena cava, the right atrium was opened from a site parallel to the atrioventricular annulus and two sutures were placed to expose the intra-atrial structure. The ASD was closed with direct 4-0 prolene sutures or with a Dacron patch. The autologous pericardium was used to repair larger ASDS with running prolene sutures. The way of deairing was the same as the TTS Group. Once the cardiopulmonary bypass ceased, the chest and pericardium are drained. The pericardium is closed and all skin incisions are closed intracutaneously (Fig. 2).

After the operation, all the patients were monitored in the intensive care unit. Bedside chest radiography was performed in the intensive care unit to exclude complications in the lungs. Mechanical ventilation was withdrawn when patients' hemodynamics became stable.

\section{Data Management and Statistical Analysis}

Nominal data are expressed as the mean \pm standard deviation (SD). Continuous variables were compared by standard t-test. Categoric variables were analyzed using chi-square analysis or the Fisher exact test. All statistical tests are two-tailed. A p-value of $<0.05$ was considered statistically significant. 


\section{Results}

\section{Demographic and clinical characteristics}

Patient characteristics and procedure-related variables were similar. Sixty-two patients (mean age: $29.37 \pm 11.6$ years) were assigned to TTS group; sixty-three patients (mean age: $24.14 \pm 15.1$ years) to RALT group. The age of patients in RALT group was younger than the patients in TTS group $(p=0.032)$. The weight in RALT group was heavier than the TTS group, and the difference was significant $(p=0.048)$. There were no significant difference between the groups in Qp/Qs and the size of defect (cm). Two groups were comparable for patient characteristics and procedure related parameters (Table 1).

\section{Peri-operative profile}

Procedures were performed successfully in all patients and no in-hospital mortality. No patient in this series was reverted to standard median sternotomy. The ASD was closed with direct 4-0 prolene sutures in 82 patients (46 cases in TTS group and 36 cases in RALT group, respectively), and the other 43 patients were closed with patch (16 cases in TTS group and 27 cases in RALT group). Immediately transesophageal echocardiographic analysis after ASD repair in all patients showed complete closure with no residual shunt. Pneumothorax occurred in six patients, two patients in TTS Group, 4 patients in RALT Group. No significant difference between the two groups $(\mathrm{p}=0.217)$. There was no significant difference in the occurrence of pulmonary infection and reoperation because of bleeding after the procedure.

As shown in Table 1, The CPB time and cross-clamp time were longer in the TTS group compared with RALT group and there were significant differences in the CPB time $(48.95 \pm 15.63 \mathrm{mins}$ vs. $31.4 \pm 8.04 \mathrm{~min}, \mathrm{p}<0.001)$ and cross-clamp time $(26.92 \pm 11.84 \mathrm{~min}$ vs. $18.51 \pm$ $6.11 \mathrm{~min}, \mathrm{p}<0.001$ ) in the TTS group compared with RALT group. Although the drainage after operation was more in the RALT group $(202.9 \pm 75.9 \mathrm{ml})$ than that in TTS group (176.5 $\pm 94.2 \mathrm{ml})$, the difference was not significant between the two groups $(p=0.087)$. The intubation time after operation was lower in TTS group (6.25 \pm $3.09 \mathrm{~h})$ compared with the RALT group (7.36 \pm 3.2$)$, and the difference was not significant $(p=0.051)$. The thoracic incision length of the procedure in RALT group $(6.02 \pm 1.03 \mathrm{~cm})$ was longer than the TTS group $(5.31 \pm$ $0.68 \mathrm{~cm})$ and the difference was significant $(\mathrm{p}<0.001)$. The ICU stays were shorter in TTS group than RALT group, and the difference was significant $(\mathrm{p}=0.032)$. The hospital stays were shorter in the TTS group compared with the RALT group, and the difference was significant $(p=0.001)$.

\section{Follow-up (patient satisfaction)}

All the patients were routinely followed-up. There were no deaths and all patients were in New York Heart Association class I with no limitation to physical activity. The overall satisfaction rate for the cosmetic results of TTS was $100 \%$ and was $96.83 \%$ (61/63 patients) in RALT patients.

The overall median satisfaction score in TTS group was 5 (range, 4-5) and was higher in the RALT group (median score, 5; range, 3-5; $\mathrm{p}<0.001$ ). All patients in TTS group were satisfied expect two patients complained the scar was too long at groin. Reasons for a lower score in RALT group included: (1) the scar in the chest was too long; (2) a RALT incision that was located too medially (coming off the bra line) and (3) asymmetrical breast development.

\section{Discussion}

The correction for atrial septal defects has changed noticeably during the past decade. Transcatheter closure of atrial septal defects has been the optimal practice and excellent results have been reported with low early and late complication rates. However, the surgical correction is still imperative for those patients who could not undergo transcatheter closure. Thus, different surgical techniques have been developed with the aim of combining good functional and cosmetic results. ${ }^{9-13)}$ Minimally invasive technique including totally thoracoscopic approach (TTS), right anterolateral thoracotomy technique (RALT), right vertical infra-axillary thoracotomy (RVIAT) is the main trend for the ASD closure to avoid the characteristic unsightly, long midline scar caused by the standard median sternotomy and gains satisfied cosmetic result.

In our department, both right anterolateral thoracotomy approach (RALT) and totally thoracoscopic approach have been used for the correction of ASD. RALT approach has been advocated for many years and has been used as a safe and cosmetic alternative to ministernotomy, ${ }^{2,13,14)}$ especially in female patients. However, this technique may impaire the breast development in female patients, particularly in the prepubescent patient. ${ }^{15)} \mathrm{We}$ have been used this procedure for several years and only 
one patients complained mild breast asymmetries. Thus, this technique is still performed in our center. Recently, totally thoracoscopic approach has been introduced to our department which has been performed to repair ASD for three years. TTS has shown some encouraging results, as in all reported cases the operation could be performed safely, and with no need for conversion to full sternotomy or minithoracotomy. ${ }^{16-19)}$ The current study provides details of early clinical results including the functional and cosmetic results from totally thoracoscopic approach and RALT in comparison.

As an alternative to standard median sternotomy, totally thoracoscopic approach to correct ASD is a well accepted technique recent years, especially accepted by the female patinets. More patients particularly female patients have underwent thoracoscopic ASD closure since early reports on endoscopic repair of congenital heart defects. Torracca and colleagues ${ }^{20)}$ reported the patients underwent successful robotic ASD closure with rapid postoperative recovery and an excellent cosmetic result compared conventional sternotomy. However, totally endoscopic techniques rely on a robotic surgical system initially. Totally thoracoscopic repair of atrial septal defect without robotic assistance is a simplified, safe and effective therapeutic option for ASD. In China, ZengShan Ma and colleagues ${ }^{21,22)}$ reported the patients underwent totally endoscopic atrial septal defect repair without robotic surgical system with excellent clinical result and gained a faster recovery of physical function and a better quality of life compared with conventional sternotomy. In our center, we performed thoracoscopic ASD closure without the robotic assistance and gained the excellent outcome just as the report. Compared with the right anterolateral thoracotomy, it is easy for the patients undergoing totally thoracoscopic repair to recover from the surgery, including ICU and hospital stay. Almost all female patients were satisfied with the procedure (Fig. 3). However, there are some disadvantages in patients undergoing totally thoracoscopic approach surgery. The curve of study was long and cardiac surgeon need more time to master the technique. The total operative time, CPB time and cross-clamp time were much longer in the totally thoracoscopic group compared with the right anterolateral thoracotomy. With the improvement of the surgeon, the operative time, CPB time and Cross-clamp time may reduce.

Right anterolateral thoracotomy approach (RALT) is another well accepted technique recent years. Several groups have reported their experiences with RALT
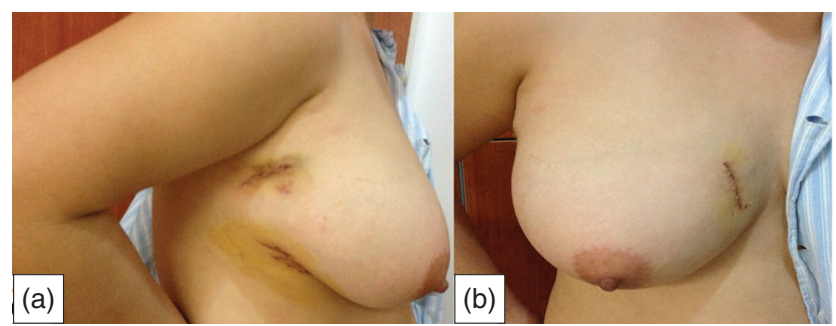

Fig. 3 The incisions of totally thoracoscopic technique after the operation: two incisions were seen in the lateral and a short incision was seen in the front.

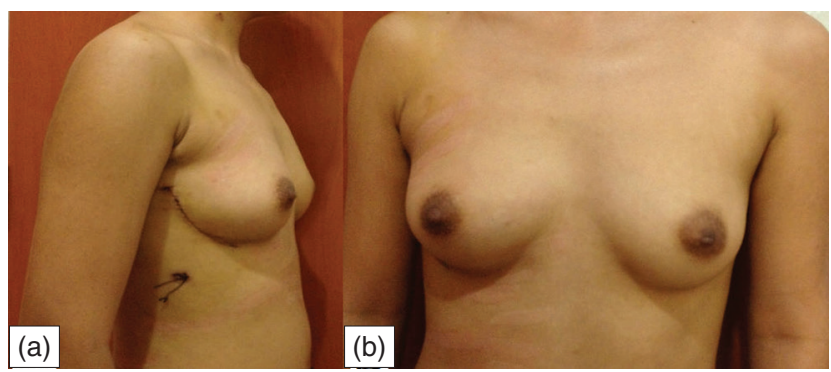

Fig. 4 The incision of right anterolateral thoracotomy technique after the procedure was seen in the lateral view and which was hided by the breast in the front.

approach as the surgical approach. Excellent results were reported for all ASD types with a right anterolateral thoracotomy approach versus standard sternotomy. ${ }^{10-13)}$ The right anterolateral thoracotomy approach has advantages compared with standard median sternotomy. The surgical scar, which is the only residue for a lifetime after the procedure, is invisible under the breast and less evident (Fig. 4). The exposition of intracardiac structures was good and the intraoperative complication rate was zero. Phrenic nerve damage, which is especially attributed to right anterolateral thoracotomy was not seen in our series. This technique was proved to be applicable to any type ASD with no operative mortality and low perioperative morbidity. Besides, the total operative time, CPB time and Cross-clamp time were not increased by this technique. The most important was that it reduced the blood transfusion and chest drainage after the procedure, the intubation time, ICU stay and hospital stay, without increasing the postoperative complications.

Both right anterolateral thoracotomy approach and totally thoracoscopic approach could repair ASD perfectly in female patients, and gain satisfied clinical and cosmetic results. Compared with the conventional surgical technique, patients can recovery quickly and improve the quality of life. The incision of both the techniques could be hidden and no significant scars compared with the conventional sternotomy. However, the length of 
incision was longer in patients undergoing right anterolateral thoracotomy approach repair than patients undergoing totally thoracoscopic repair for ASD, especially in adult patients. Recently, CPB was established by peripheral arterial and venous cannulating, which significantly shortened the incision. In younger patients, CPB could not be established by peripheral arterial and venous cannulating and the incision was not short, which may lead to unsatisfied procedure. Totally thoracoscopic approach surgery could address the problem, which dispersed the incisions and the scars were not significant even in the front. As the improvement of surgeons, the incision could shorten and gain more cosmetic results.

According to our results, almost all the female patients were satisfied with the totally thoracoscopic approach and only several patients were unsatisfied with the right anterolateral thoracotomy approach. Only two patients complained the long scar and keloid at the incision level. During the follow-up, the satisfaction store of TTS patient was higher than RALT patient. Two patients in TTS group complained the scar was too long at groin. As the development of surgery, the CPB may be established by the percutaneous arterial and venous cannulating, which avoid the incision at groin. The reasons for a lower score in RALT group included: (1) the scar in the chest was too long; (2) a RALT incision that was located too medially (coming off the bra line) and (3) asymmetrical breast development. The incidence of asymmetrical breast development was lower than the report. ${ }^{23)}$ If the CPB can be established by the peripheral arterial and venous cannulating, the incision in the chest may be shorter. Attention should be paied to the incision, which should be away from the breast tissue and the pectoralis major muscle to avoid the normal breast development.

There were some limitations in this study. It was a retrospective data analysis comparing two different minimally invasive approaches to correct ASD in female patients in our institution. The patients enrolled had a shorter follow-up. We did not have the strict criterion to evaluate the asymmetrical breast development. The asymmetrical breast development was supposed by the patients, which may confuse the incidence of asymmetrical breast development.

In conclusion, both TTS and RALT are valid and reliable cosmetic surgical techniques for repairing ASDs in female patients. Both techniques allow excellent cosmetic and functional results in most female patients. The totally thoracoscopic technique may gain shorter incision and cosmetic results compared with RALT.

\section{Disclosure Statements}

None.

\section{Reference}

1) Chessa M, Carminati M, Butera G, et al. Early and late complications associated with transcatheter occlusion of secundum atrial septal defect. J Am Coll Cardiol 2002; 39: 1061-5.

2) Vida VL, Padalino MA, Motta R, et al. Minimally invasive surgical options in pediatric heart surgery. Expert Rev Cardiovasc Ther 2011; 9: 763-9.

3) Aybek T, Dogan S, Risteski PS, et al. Two hundred forty minimally invasive mitral operations through right minithoracotomy. Ann Thorac Surg 2006; 81: 1618-24.

4) McClure RS, Cohn LH, Wiegerinck E, et al. Early and late outcomes in minimally invasive mitral valve repair: an eleven-year experience in 707 patients. J Thorac Cardiovasc Surg 2009; 137: 70-5.

5) Liang T, XiangJun Z, XiaoJing M, et al. New minimally invasive technique to occlude secundum atrial septal defect in 53 patients. Ann Thorac Surg 2006; 81: $1417-9$.

6) Hongxin L, Wenbin G, Lijun S, et al. Intraoperative device closure of secundum atrial septal defect with a right anterior minithoracotomy in 100 patients. J Thorac Cardiovasc Surg 2007; 134: 946-51.

7) Reichenspurner H, Detter C, Deuse T, et al. Video and robotic-assisted minimally invasive mitral valve surgery: a comparison of the Port-Access and transthoracic clamp techniques. Ann Thorac Surg 2005; 79: 485-90; discussion 490-1.

8) Vida VL, Padalino MA, Boccuzzo G, et al. Minimally invasive operation for congenital heart disease: a sex-differentiated approach. J Thorac Cardiovasc Surg 2009; 138: 933-6.

9) Hagl C, Stock U, Haverich A, et al. Evaluation of different minimally invasive techniques in pediatric cardiac surgery: is a full sternotomy always a necessity? Chest 2001; 119: 622-7.

10) Abdel-Rahman U, Wimmer-Greinecker G, Matheis G, et al. Correction of simple congenital heart defects in infants and children through a minithoracotomy. Ann Thorac Surg 2001; 72: 1645-9.

11) De Mulder W, Vanermen H. Repair of atrial septal defects via limited right anterolateral thoracotomy. Acta Chir Belg 2002; 102: 450-4.

12) Mishaly D, Ghosh P, Preisman S. Minimally invasive congenital cardiac surgery through right anterior minithoracotomy approach. Ann Thorac Surg 2008; 85: 831-5.

13) Däbritz S, Sachweh J, Walter M, et al. Closure of atrial septal defects via limited right anterolateral thoracotomy as a minimal invasive approach in female patients. Eur J Cardiothor Surg 1999; 15: 18-23. 
14) Lancaster LL, Mavroudis C, Rees AH, et al. Surgical approach to atrial septal defect in the female. Right thoracotomy versus sternotomy. Am Surg 1990; 56: $218-21$.

15) Bleiziffer S, Schreiber C, Burgkart R, et al. The influence of right anterolateral thoracotomy in prepubescent female patients on late breast development and on the incidence of scoliosis. J Thorac Cardiovasc Surg 2004; 127: 1474-80.

16) Wimmer-Greinecker G, Dogan S, Aybek T, et al. Totally endoscopic atrial septal repair in adults with computer-enhanced telemanipulation. J Thorac Cardiovasc Surg 2003; 126: 465-8.

17) Argenziano $\mathrm{M}, \mathrm{Oz} \mathrm{MC}$, Kohmoto $\mathrm{T}$, et al. Totally endoscopic atrial septal defect repair with robotic assistance. Circulation 2003; 108: II191-4.

18) Bonaros N, Schachner T, Oehlinger A, et al. Robotically assisted totally endoscopic atrial septal defect repair: insights from operative times, learning curves, and clinical outcome. Ann Thorac Surg 2006; 82: 687-93.
19) Morgan JA, Peacock JC, Kohmoto T, et al. Robotic techniques improve quality of life in patients undergoing atrial septal defect repair. Ann Thorac Surg 2004; 77: 1328-33.

20) Torracca L, Ismeno G, Alfieri O. Totally endoscopic computer-enhanced atrial septal defect closure in six patients. Ann Thorac Surg 2001; 72: 1354-7.

21) Ma ZS, Dong MF, Yin QY, et al. Totally thoracoscopic repair of atrial septal defect without robotic assistance: a single-center experience. J Thorac Cardiovasc Surg 2011; 141: 1380-3.

22) Ma ZS, Yin QY, Dong MF, et al. Quality of life in patients undergoing totally thoracoscopic closure for atrial septal defect. Ann Thorac Surg 2011; 92: 2230-4.

23) Vida VL, Tessari C, Fabozzo A, et al. The evolution of the right anterolateral thoracotomy technique for correction of atrial septal defects: cosmetic and functional results in prepubescent patients. Ann Thorac Surg 2013; 95: 242-7. 